\title{
In vitro Replication Activity of Bovine Viral Diarrhea Virus in an Epithelial Cell Line and in Bovine Peripheral Blood Mononuclear Cells
}

\author{
Lauretta TURIN ${ }^{1)}$, Barbara LUCCHINI ${ }^{1)}$, Valerio BRONZO ${ }^{1)}$ and Camilla LUZZAGO ${ }^{1 *}$ \\ ${ }^{1)}$ Department of Veterinary Pathology, Hygiene and Public Health, University of Milan, Via Celoria 10, 20133 Milano, Italy
}

(Received 12 January 2012/Accepted 29 May 2012/Published online in J-STAGE 12 June 2012)

\begin{abstract}
The present study focused on the in vitro infection of Madin-Darby bovine kidney (MDBK) cells and bovine peripheral blood mononuclear cells (PBMCs) from naïve animals with non-cytopathic (ncp, BVDV-1b NY-1) and cytopathic (cp, BVDV-1a NADL) strains. Infections with 0.1 and 1 multiplicity of infections (MOI) and incubation times of 18 and $36 \mathrm{hr}$ were compared. Twelve BVDV naïve heifers were enrolled to collect PBMCs. The viral loads in MDBK cells and in PBMCs after in vitro infections were measured by real-time polymerase chain reaction (PCR) assays. The highest viral loads were measured at $1 \mathrm{MOI}$ and $36 \mathrm{hr}$ post infection in both cell systems and the lowest at $0.1 \mathrm{MOI}$ and $18 \mathrm{hr}$ with the exception of the cp strain NADL in PBMCs, for which the highest viral load was observed at $0.1 \mathrm{MOI}$ and $36 \mathrm{hr}$. Viral load mean values were higher for the cp strain than the ncp strain irrespective of the extent of the infection period and MOI. The models of infection studied uncovered different replication activities respectively according to the biotype of virus, the cell substrate and the duration of infection. Replication tends to be higher in PBMCs, particularly at low MOIs and for the ncp strain.

KEY WORDS: bovine viral diarrhea virus, in vitro infection, MDBK, PBMC, real-time PCR.
\end{abstract}

doi: 10.1292/jvms.12-0011; J. Vet. Med. Sci. 74(11): 1397-1400, 2012

Bovine viral diarrhea virus (BVDV) is an economically important pathogen of cattle belonging to the Pestivirus genus of the Flaviviridae family. Genetic typing of BVDV isolates has distinguished two species, BVDV-1 and BVDV-2, plus a recent putative BVDV-3 species [11]. While the former virus species includes at least 11 subtypes, BVDV-1a to $-1 \mathrm{k}$ [19], BVDV-2 viruses are classified into two subtypes [15].

A number of studies are available on in vivo experimental infections, while the in vitro models used to investigate the infectivity and replication of different BVDV genotypes and subtypes in permissive cells are limited $[2,16]$. Therefore, the development of in vitro systems is a valid support to underlie the interactions of different virus subtypes with cell substrates and correlate them with the in vivo infection. This objective is particularly important because a wide spectrum of virulence has been observed among strains belonging to the same genotype, such as that of the well known BVDV2 species [8, 10]. Recently, a virulence variation among BVDV-1 subtypes has been reported based on comparison of clinical outcomes following in vivo infection [17].

BVDV infects a wide variety of cell types but has a preferential tropism for immune cells, affecting their functions. BVDV infects both cells of the innate arm, such as neutrophils, monocytes, macrophages and dendritic cells, and cells devoted to the adaptive response, namely T- and B-lymphocytes [7, 18].

\footnotetext{
*Correspondence to: Luzzago, C., Department of Veterinary Pathology, Hygiene and Public Health, University of Milan, Via Celoria 10, 20133 Milano, Italy.

e-mail: camilla.luzzago@unimi.it

(C)2012 The Japanese Society of Veterinary Science
}

Following in vitro and in vivo infections, both noncytopathic (ncp) and cytopathic (cp) biotypes can affect the activity of alveolar macrophages; alterations of the surface expression of the Fc receptor and the complement receptor result in depressed phagocyte activity, impairment of the microbicidal activity and reduced responsiveness to chemotactic factors $[4,14]$.

The aim of the present study was to investigate the in vitro replication activity of BVDV-1 $\mathrm{cp}$ and ncp biotypes in Madin-Darby bovine kidney (MDBK) cells and peripheral blood mononuclear cells (PBMCs) from BVDV naïve animals.

\section{MATERIALS AND METHODS}

Animal selection and blood handling: This study involved 12 BVDV naïve Holstein-Friesian heifers (10-14 months old) from three BVDV-free dairy herds that participated in a BVDV control program [12]. None of the animals had received vaccination against BVDV. The experimental protocol was ethically approved by the Italian Ministry of Health (protocol number 2006070977-003).

Blood samples from each animal were collected with and without anticoagulant (lithium-heparin), kept at $4^{\circ} \mathrm{C}$ during delivery to the laboratory and processed within $2 \mathrm{hr}$. Sera were isolated from blood samples without anticoagulant by centrifugation at $600 \times \mathrm{g}$ for $10 \mathrm{~min}$ and stored at $-20^{\circ} \mathrm{C}$.

Sera from each animal were tested by virus neutralization (VN) test [6] for the presence of BVDV antibodies against different BVDV-1 and BVDV-2 strains.

PBMCs from each animal were separated by density gradient centrifugation from blood with anticoagulant; after centrifugation at $1200 \times g$ for $10 \mathrm{~min}$, the buffy coat was collected, diluted 1:2 with phosphate buffered saline (PBS) 
and stratified on Histopaque-1077 (Sigma-Aldrich, Munich, Germany) at a the proportion of $2 / 3$. After centrifugation at $400 \times g$ for $30 \mathrm{~min}$ at room temperature, PBMCs were collected and washed three times in PBS. Viable PBMCs were counted (trypan blue exclusion method) and resuspended at $6 \times 10^{6}$ cells $/ \mathrm{m} l$ in RPMI-1640 medium supplemented with $10 \%$ BVDV- and antibody-free fetal calf serum (FCS), $1 \%$ L-glutamine and $0.01 \% \beta$-mercaptoethanol.

Viruses and cells: Two viral strains were used for in vitro infections, BVDV-1a cp NADL (ATCC VR-534) and BVDV-1b ncp NY-1 (ATCC VR-524); the BVDV-1a cp Singer strain (NVSL, Ames, IA, U.S.A.) was used as a calibrator for real-time polymerase chain reaction (PCR) quantification. To obtain the stocks, the viruses were grown in MDBK cells (ATCC CCL-22), maintained in minimal essential medium (MEM) with 10\% FCS, 1\% L-glutamine 200 $\mathrm{mM}$ and $100 \mathrm{U} / \mathrm{m} l$ penicillin, $100 \mu \mathrm{g} / \mathrm{m} l$ streptomycin and $2.5 \mu \mathrm{g} / \mathrm{m} l$ fungizone. The MDBK cell line was also used for virus stocks titration, serological tests and in vitro infections.

Virus titration: Tissue culture end point titration in 96well plates was performed for quantification of each virus stock; tenfold dilutions of each virus stock in quadruplicate were mixed with a suspension of $1.5 \times 10^{4} \mathrm{MDBK}$ cells and incubated at $37^{\circ} \mathrm{C}$ in $5 \% \mathrm{CO}_{2}$ for 4 days. The infectivity for the cp strains was detected by observation of cytopathic effects (CPE). For the ncp strains, infectivity was determined by immunoperoxidase monolayer assay (IPMA) [13]; monoclonal antibody 20.10.06 was chosen because of its broad cross-reactivity [5]. The development of red cytoplasmic staining in at least one cluster of infected cells was indicative of a positive reaction. For both ncp and cp strains, the virus titer was expressed by $50 \%$ tissue culture infective dose $/ \mu l$ $\left(\mathrm{TCID}_{50} / \mu l\right)$, according to the Spearman-Karber method.

$M D B K$ and PBMCs infections: MDBK cells, suspended in the amount of $10^{5} /$ well in culture medium, were seeded in 24-well plates and allowed to adhere for $2 \mathrm{hr}$ at $37^{\circ} \mathrm{C}$ in $5 \%$ $\mathrm{CO}_{2}$. Infections with each viral strain at 0.1 and at $1 \mathrm{MOI}$ were performed in 4 wells, and an extra well was used as a negative control. Viruses were allowed to adsorb for $1 \mathrm{hr}$ at $37^{\circ} \mathrm{C}$ in $5 \% \mathrm{CO}_{2}$. After this period, non-adsorbed viruses were eliminated by washing MDBK monolayer cells twice with culture medium. The plates were incubated at $37^{\circ} \mathrm{C}$ in $5 \% \mathrm{CO}_{2}$, for 18 and $36 \mathrm{hr}$. MDBK assays were repeated twice.

PBMCs obtained from each animal as described above, suspended in the amount of $1.2 \times 10^{6} /$ well in PBMC culture medium, were seeded in $24-w e l l$ plates. Five replicates were seeded for each assay, the negative control and 4 wells for infection with each viral strain at 0.1 and at $1 \mathrm{MOI}$, allowing an adsorption time of $1 \mathrm{hr}$ at $37^{\circ} \mathrm{C}$ in $5 \% \mathrm{CO}_{2}$. After this period, non-adsorbed virus was eliminated by cell washing and centrifugation twice in PBMC culture medium. The cell pellets were resuspended in PBMC culture medium, plated and incubated at $37^{\circ} \mathrm{C}$ in $5 \% \mathrm{CO}_{2}$ for 18 and $36 \mathrm{hr}$.

After incubation, both MDBK and PBMC plates were frozen at $-80^{\circ} \mathrm{C}$ until virus quantification was performed.

Quantification of viral loads by real-time PCR: The total content of each well, MDBK cells plus culture medium and
PBMCs plus culture medium, underwent viral RNA extraction with a QIAamp Viral RNA Mini Kit (Qiagen, Hilden, Germany); the final concentration of RNA was determined using a spectrophotometer at a wavelength of $260 \mathrm{~nm}$. The whole sample, corresponding to about $1 \mu \mathrm{g}$ of RNA, was reverse transcribed to cDNA using a high-capacity cDNA archive retrotranscription kit with random hexamers (Applied Biosystems, Foster City, CA, U.S.A.). For the calibrator, BVDV-1a Singer strain RNA was extracted with the same protocol from the supernatant of MDBK cells containing $2000 \mathrm{TCID}_{50} / \mu l$. The cDNA $(9 \mu l)$ obtained from the reference virus and from each sample was used as a template for TaqMan real-time PCR assays in an optimized reaction volume of $20 \mu \mathrm{l}$ on MicroAmp optical 96-well plates in the presence of 2X Master Mix (10 $\mu l)$ (Applied Biosystems), probe and $300 \mathrm{nM}$ each of the primers $(0.3 \mu \mathrm{l}$ of $10 \mu \mathrm{M}$ solution) [3]. In addition to cDNA samples, duplicates of a 10 -fold dilution of cDNA from the reference virus were included in each plate and used to generate a calibration curve for virus quantification. Preliminarily, 10-fold serial dilutions of reference virus cDNA were used as templates to generate standard curves for determination of the assay sensitivity. A duplicate no-template control was also included in each plate. Real-time quantitative PCR was carried out in an ABI PRISM 7000 Sequence Detection System, and results were analyzed with its software (Applied Biosystems). For each sample tested, the virus quantity was calculated in relationship to the viral strain calibrator and expressed as the $\mathrm{TCID}_{50}$ /assay.

\section{RESULTS}

The virus quantification in the present study was performed on the total volume of cells and medium in order to make sure to record all of the virus. Indeed, we had performed preliminary assays (data not shown) comparing the cells alone versus the medium alone and versus the cells plus the medium. The results of the preliminary assays showed positive signals for virus in all the samples, with a higher amount of virus detected in cells plus medium than in cells or medium separately. Therefore, we decided to quantify the medium and cells together.

The results obtained after infection of MDBK cells with NADL and NY-1 BVDV strains showed the highest viral loads for both strains at $1 \mathrm{MOI}$ and $36 \mathrm{hr}$ post infection. Higher viral loads were observed for the cp strain than the ncp strain. This difference was observed irrespectively of the extent of the infection period and MOI. In particular, NADL and NY-1 reached mean values ( \pm standard error) of 61.94 $( \pm 45.28)$ and $35.03( \pm 31.90) \mathrm{TCID}_{50} /$ assay respectively at 1 MOI and $36 \mathrm{hr}$ versus $4.69( \pm 1.97)$ and $2.84( \pm 2.83) \mathrm{TCID}_{50} /$ assay at $1 \mathrm{MOI}$ and $18 \mathrm{hr}$. For NY-1, $0.1 \mathrm{MOI}$ showed slightly positive results both at $18(0.14 \pm 0.03)$ and $36(0.49 \pm 0.39)$ hr. The results are summarized in Fig. 1.

PBMCs from all tested animals were infected by the selected BVDV strains at both times of infection and MOIs. All the selected animals were negative for $\mathrm{VN}$ antibodies against BVDV-1 and -2 strains at the time of the blood sam- 


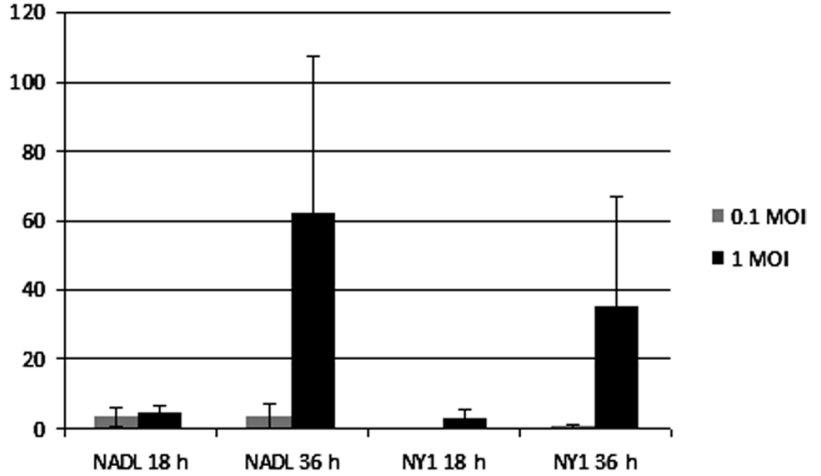

Fig. 1. Comparison of viral loads in an MDBK cell line following in vitro infection.

MDBK cells were infected with 0.1 and 1 MOI of BVDV strains and collected at $18 \mathrm{hr}$ and $36 \mathrm{hr}$ post infection. Data are reported as mean values ( \pm standard error). The virus quantity was calculated in relationship to the BVDV calibrator and expressed as $\mathrm{TCID}_{50} /$ assay.

pling. Infected PBMCs showed higher viral loads for the $\mathrm{cp}$ strain than the ncp strain. This difference was observed at both times of infection and MOIs with the exception of 1 MOI at $36 \mathrm{hr}$. In this case, the viral load of NY-1 was 28.41 $( \pm 8.73) \mathrm{TCID}_{50} /$ assay versus $21.15( \pm 2.94) \mathrm{TCID}_{50} /$ assay for NADL. The results are summarized in Fig. 2.

When comparing the 2 in vitro models, an opposite trend was observed at $1 \mathrm{MOI}$ for NADL strains in MDBK cells versus PBMCs in terms of the amount of virus, which increased in MDBK cells and decreased in PBMCs.

\section{DISCUSSION}

The in vitro permissivity of MDBK cells and PBMCs from BVDV naïve animals to infection with 0.1 or 1 MOIs BVDV-1 reference strains was investigated by mean of quantification of viral loads at 18 and $36 \mathrm{hr}$ post infection.

MDBK cells and PBMCs were efficiently infected by NADL and NY-1 strains at both times of infection and MOIs. The highest viral loads were measured at $1 \mathrm{MOI}$ and $36 \mathrm{hr}$ post infection in both cell systems with the exception of the cp strain NADL in PBMCs, which allowed an intense replication rate already at $18 \mathrm{hr}$ at both MOIs. Indeed, PBMCs represent, although in an in vitro system, the main target for BVDV proliferation, as observed for both the strains investigated. The opposite trend observed for NADL at $36 \mathrm{hr}$ in the 2 models can be explained by the different cell substrates available for the virus. Indeed, PBMCs do not replicate in vitro, while MDBK cells are characterized by a high replication rate. Therefore, we could hypothesize that the $\mathrm{cp}$ virus at the higher post infection time and higher MOI causes damage to all the available substrates necessary for replication, as supported by the efficient growth for the ncp strain, that does not affect the cells. Since the progression of infection up to $36 \mathrm{hr}$ seems to be affected by PBMC vitality, a kinetic study in a narrow range of time post infection would be useful to highlight virus replication properties of different

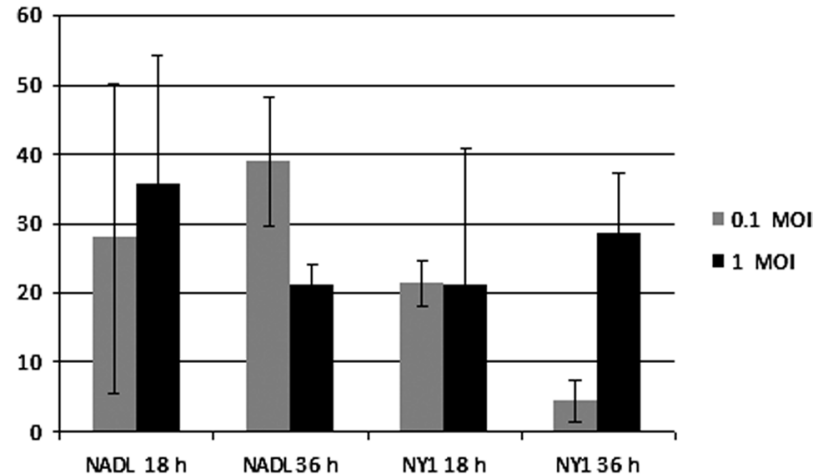

Fig. 2. Comparison of viral loads in PBMCs from 12 BVDV naïve heifers following in vitro infection. PBMCs were infected with 0.1 and $1 \mathrm{MOI}$ of BVDV strains and collected at $18 \mathrm{hr}$ and $36 \mathrm{hr}$ post infection. Data are reported as mean values ( \pm standard error). The virus quantity was calculated in relationship to the BVDV calibrator and expressed as $\mathrm{TCID}_{50}$ /assay.

\section{BVDV strains.}

Concerning the different replication activities of BVDV biotypes, we observed higher viral loads for cp strains than ncp strains in both cell types. Our results are in accordance with other reports, which showed an increase of virus replication and production of a large amount of dsRNA in cells infected with cp, but not with ncp BVDV strains $[1,9,20]$. This feature may represent a survival strategy of ncp strains, because low production of viral particles can prove to be helpful in escaping the immune system and consequently persist in the host population.

Comparison of $\mathrm{cp}$ and ncp homologous biotypes could determine whether such difference is due to the strain itself or to the biotype.

In conclusion, PBMCs, which were efficiently infected in all the tested conditions, were shown to be a fitting substrate mainly for the ncp strain. This preliminary result shows that such an infection model could be useful to investigate the virus-host interactions of different subtypes and to correlate them with in vivo infection without any ethical impact and at lower costs. A wider panel of ncp BVDV strains characterized by a different in vivo virulence profile would be required to confirm the trends that we preliminarily observed.

ACKNOWLEDGMENTS. We are very grateful to Prof. Wilma Ponti for the valuable contribution to the experimental design and for helpful discussions. We acknowledge Professor E. J. Dubovi for kindly providing monoclonal antibody. Further, we wish to thank Dr. Michela Frigerio for help in collecting samples. This work was financially supported by a PRIN Grant (2006) from the Italian Ministry of Education, Scientific Research and Health.

\section{REFERENCES}

1. Becher, P., Orlich, M. and Thiel, H. J. 2001. RNA recombination between persisting pestivirus and a vaccine strain: generation of 
cytopathogenic virus and induction of lethal disease. J. Virol. 75: 6256-6264. [Medline] [CrossRef]

2. Beer, M., Wolf, G., Pichler, J., Wolfmeyer, A. and Kaaden, O. R. 1997. Cytotoxic T-lymphocyte responses in cattle infected with bovine viral diarrhea virus. Vet. Microbiol. 58: 9-22. [Medline] [CrossRef]

3. Bhudevi, B. and Weinstock, D. 2001. Fluorogenic RT-PCR assay (TaqMan) for detection and classification of bovine viral diarrhea virus. Vet. Microbiol. 83: 1-10. [Medline] [CrossRef]

4. Chase, C. C., Elmowalid, G. and Yousif, A. A. 2004. The immune response to bovine viral diarrhea virus: a constantly changing picture. Vet. Clin. North Am. Food Anim. Pract. 20: 95-114. [Medline] [CrossRef]

5. Corapi, W. V., Donis, R. O. and Dubovi, E. J. 1990. Characterization of a panel of monoclonal antibodies and their use in the study of the antigenic diversity of bovine viral diarrhea virus. Am. J. Vet. Res. 51: 1388-1394. [Medline]

6. Edwards, S. 1990. The diagnosis of bovine virus diarrhea-mucosal disease in cattle. Rev. Sci. Tech. Off. Int. Epiz. 9: 115-130.

7. Glew, E. J., Carr, B. V., Brackenbury, L. S., Hope, J. C., Charleston, B. and Howard, C. J. 2003. Differential effects of bovine viral diarrhoea virus on monocytes and dendritic cells. J. Gen. Virol. 84: 1771-1780. [Medline] [CrossRef]

8. Kelling, C. L., Steffen, D. J., Topliff, C. L., Eskridge, K. M., Donis, R. O. and Higuchi, D. S. 2002. Comparative virulence of isolates of bovine viral diarrhea virus type II in experimentally inoculated six-to nine-month-old calves. Am. J. Vet. Res. 63: 1379-1384. [Medline] [CrossRef]

9. Lackner, T., Müller, A., Pankraz, A., Becher, P., Thiel, H. J., Gorbalenya, A. E. and Tautz, N. 2004. Temporal modulation of an autoprotease is crucial for replication and pathogenicity of an RNA virus. J. Virol. 78: 10765-10775. [Medline] [CrossRef]

10. Liebler-Tenorio, E. M., Ridpath, J. F. and Neill, J. D. 2003. Distribution of viral antigen and development of lesions after experimental infection of calves with a BVDV 2 strain of low virulence. J. Vet. Diagn. Invest. 15: 221-232. [Medline] [CrossRef]

11. Liu, L., Xia, H., Wahlberg, N., Belák, S. and Baule, C. 2009.
Phylogeny, classification and 166 evolutionary insights into pestiviruses. Virology 385: 351-357. [Medline] [CrossRef]

12. Luzzago, C., Frigerio, M., Piccinini, R., Daprà, V. and Zecconi, A. 2008. A scoring system for risk assessment of the introduction and spread of bovine viral diarrhoea virus in dairy herds in Northern Italy. Vet. J. 177: 236-241. [Medline] [CrossRef]

13. Meyling, A. 1984. Detection of BVD virus in viraemic cattle by an indirect immunoperoxidase technique. pp. 37-46. In: Recent Advances in Virus Diagnosis (CEC Seminar). (McNulty, M.S. and McFerran, J.B. eds.), Martinus Nijhoff, Belfast.

14. Peterhans, E., Jungi, T. W. and Schweizer, M. 2003. BVDV and innate immunity. Biologicals 31: 107-112. [Medline] [CrossRef]

15. Ridpath, J. F., Neill, J. D., Frey, M. and Landgraf, J. G. 2000. Phylogenetic, antigenic and clinical characterization of type 2 BVDV from North America. Vet. Microbiol. 77: 145-155. [Medline] [CrossRef]

16. Ridpath, J. F., Bendfeldt, S., Neill, J. D. and Liebler-Tenorio, E. 2006. Lymphocytopathogenic activity in vitro correlates with high virulence in vivo for BVDV type 2 strains: criteria for a third biotype of BVDV. Virus Res. 118: 62-69. [Medline] [CrossRef]

17. Ridpath, J. F., Neill, J. D. and Peterhans, E. 2007. Impact of variation in acute virulence of BVDV1 strains on design of better vaccine efficacy challenge models. Vaccine 25: 8058-8066. [Medline] [CrossRef]

18. Sopp, P., Hooper, L. B., Clarke, M. C., Howard, C. J. and Brownlie, J. 1994. Detection of bovine viral diarrhoea virus p80 protein in subpopulations of bovine leukocytes. J. Gen. Virol. 75: 1189-1194. [Medline] [CrossRef]

19. Vilček, S., Ďurkovič, B., Kolesárová, M., Greiser-Wilke, I. and Paton, D. 2004. Genetic diversity of international bovine viral diarrhea virus (BVDV) isolates: identification of a new BVDV-1 genetic group. Vet. Res. 35: 609-615. [Medline] [CrossRef]

20. Yamane, D., Kato, K., Tohya, Y. and Akashi, H. 2006. The double-stranded RNA-induced apoptosis pathway is involved in the cytopathogenicity of cytopathogenic Bovine viral diarrhea virus. J. Gen. Virol. 87: 2961-2970. [Medline] [CrossRef] 